\title{
Development of Low Cost Microcontroller based Pulse Electro- Magnetic Pulse Therapy System for Pain relief and Bone Healing
}

\author{
Joyanta Kumar Roy ${ }^{1}$, Joydeep Roy ${ }^{2}$ \\ ${ }^{I}$ Dean (Research \& Consultancy), Professor Head, Department of Electronics and Communication \\ Engineering, MCKV Institute of Engineering, W.B. India \\ ${ }^{2}$ Project Engineer, Wipro Tech, India.
}

\begin{abstract}
Pulse Electromagnetic therapy is usually referred by doctors for clinical management of quick bone healing, repair of damaged tissues and as a quick remedy for pain. Various PEMT equipment are already available in market, but none of them are available as low cost consumer electronic device. Authors tried to develop a low cost version of micro controller controlled PEMT device. The design and construction method of this PEMT device has been thoroughly discussed in this paper. The prototype constructed not only met the low cost criteria but also was equally effective at pain management and bone healing.
\end{abstract}

Keywords: Pulse electromagnetic field, PEMF therapy, Microcontroller, Electro- magnetic coil, Tissue Healing

\section{Introduction}

Pulse Electromagnetic Therapy (PEMFT), also called magneto therapy (PEMF) is one of the common reparative techniques used by orthopedics to treat non-union fracture, failed fusion, congenital pseudarthrosis and depression. It is also used to fasten healing of ligaments and to ease pain. In the case of bone healing, PEMF uses electrical energy to direct a series of magnetic pulses through injured tissue whereby each magnetic pulse induces a tiny electrical signal that stimulates cellular repair.

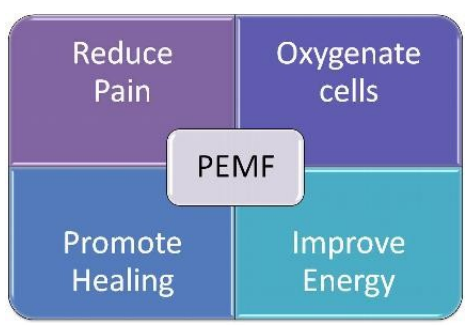

Figure 1: Roles of PEMF

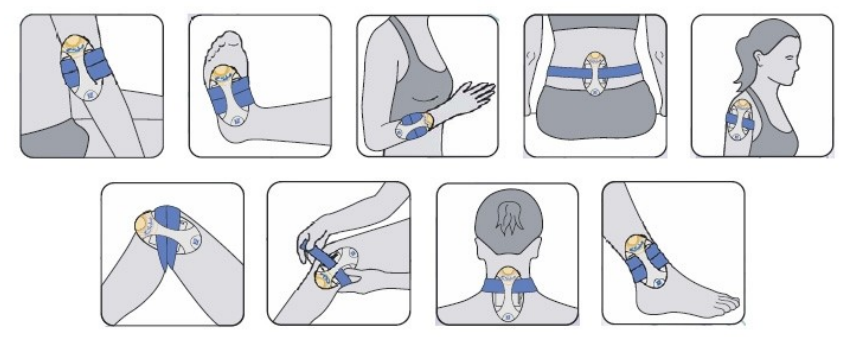

Figure 2: PEMF application locations for pain management

Many studies have proved the effectiveness of PEMF in healing soft-tissues wounds also. It is also known to suppress inflammatory responses and thus ameliorate pain. PEMF reduces pain, oxygenates cells, promotes healing and improves energy. Frequency specific pulsed electro-magnetic fields are the most effective and also the most cost efficient. The magnetic field of 30-60 Micro Tesla is found to be the useful intensity range of PEMF and $0-30 \mathrm{~Hz}$ is found to be the useful frequency. Studies show, a frequency of $2 \mathrm{~Hz}$ promotes Nerve regeneration, a frequency of $7 \mathrm{~Hz}$ fastens bone healing, whereas a frequency of $10 \mathrm{~Hz}$ helps in ligament healing. A frequency of $15-20 \mathrm{~Hz}$ stimulates capillary formation.

PEMF provides excellent regenerative effects because the magnetic field helps the cells to regenerate naturally, and thus optimizes the body's ability to heal. A magnetic switch also increases the cellular energy production, reducing oxidation level in the process. 


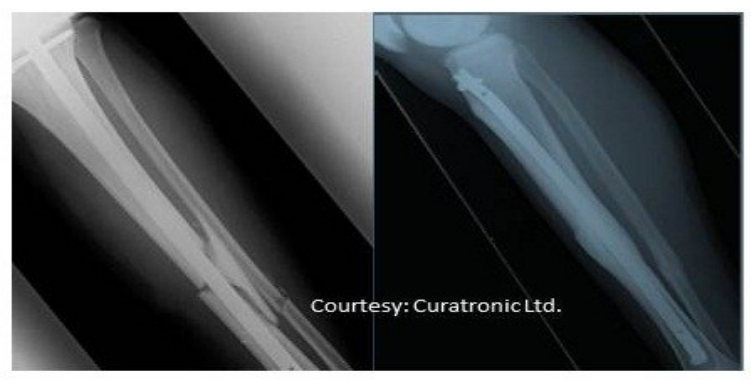

a) Fractured leg after surgery

b) Healed leg After PEMFT treatment

Figure 3: Fractured leg after surgery and healed leg after PEMFT

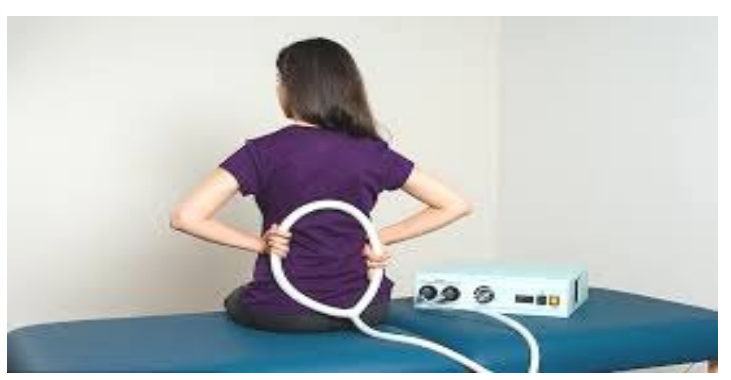

Figure 4: PEMF treatment for low back pain

A magnetic field also affects the charge of the cell membrane, causing membrane channels to open up and thus allowing more nutrients to reach to the cells and at the same time easing the process of releasing waste materials. This helps restore and rebalance healthy cell function. Magnetic fields protect against cell injury by improving circulation, repair processes and energy, and increasing special stress proteins in the cells. These proteins are used to prevent cell breakdown and wear and tear as well as helps in speedy recovery from injury (Fig1). Magnetic fields balance cells, tissues and bodily functions at very fundamental levels much before any damage takes place.

Bassett CA, et al. used PEMF therapy [2] for the treatment of hip having femoral head osteonecrosis on 118 patient found long term improvement in symptoms and signs together with reduced rate of early joint arthoplasty. Figure -3 is the clinical example of bone healing before and after EMPF therapy. Markos, Markov discusses [3] the uses of magnetic and electromagnetic field successful use a modern medicine. He mentioned that PEMF therapy is very useful for the treatment of musculoskeletal disorder and pain. It was also found in literature survey [4] that PEMF is non-invasive and cost effective adjuvant treatment in experimental osteochondral defect healing. Dr. Sircus [5] posted the facts of pulse electromagnetic field therapy for relieving the pain of cancer. Walter H. Chang, et al. [7] in his extensive research found that using pulse electromagnetic Bone defect healing can be enhance by Pulse electromagnetic therapy.

Fig. 3 is an example of bone healing after application of PEMF therapy on leg fracture. There are many applications in EMPF therapy at various parts of the body as shown in Fig.-2. The fig.4 is depicting the application of PEMF in back pain application.

\section{System Architecture And Proposed Methodology}

\subsection{Project Objectives}

The issue of pain treatment is an extremely urgent health and socio-economic problem. Pain, in acute, recurrent and chronic forms, is prevalent across age, cultural background, and sex, and costs North American adults an estimated $\$ 10,000$ to $\$ 15,000$ per person annually. The cost medical treatment is very high in European countries also. So if a low cost portable electromagnetic pulse therapy instrument can be developed then it will reduce the cost of pain management and will play as alternative medicine.

- The equipment should be user friendly. The device which helps in relief of muscular pain and quick healing of wounds and bone injuries.

- The therapy helps in quick healing, low-cost, user-friendly and effective solution of wounds for people of all ages. Also it has no side effects as compared to conventional medicine

- The device will be made off with low cost components and will be programmable for electrical aspects relating to bone healing and pain management. 


\subsection{HARDWARE DESIGN}

\subsubsection{DESIGN OF MAGNETIC COIL}

We are using an air core electromagnetic solenoid as our magnetic coil. Fig. 5 shows the cross section of magnetic coil.

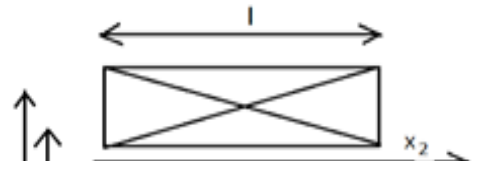

Figure 5: Cross section of magnetic coil

The equation of magnetic flux at any point $\mathrm{B}$ in general case is given by below equation:

Where:

$$
\mathbf{B}=\frac{\left(\mu_{0} * i * n\right)}{2\left(r_{2}-r_{1}\right)}\left[x_{2} \ln \frac{\sqrt{\left(r_{2}^{2}-x_{2}^{2}\right)}+r_{2}}{\sqrt{\left(r_{1}^{2}-x_{2}^{2}\right)}+r_{1}}-x_{1} \ln \frac{\sqrt{\left(r_{2}^{2}-x_{1}^{2}\right)}+r_{2}}{\sqrt{\left(r_{1}^{2}-x_{1}^{2}\right)}+r_{1}}\right]
$$

id. The direction of field

\section{cross secuon or pnysical coll}

$\mu_{0}=\quad$ Permeability constant $(1.26 * 10-6 \mathrm{Tm} / \mathrm{A}$, for coil measure in meter $)$

$\mathrm{i}=$ The current in the wire, in amperes.

$\mathrm{n}=\quad$ The number of turns of wire per unit length in the solenoid.

r1 $=\quad$ The inside radius of solenoid.

$\mathrm{r} 2=$ The outside radius of solenoid.

$\mathrm{x} 1$ and $\mathrm{x} 2$ are the distances, on axis from the end of the solenoid to the magnetic field measurement point

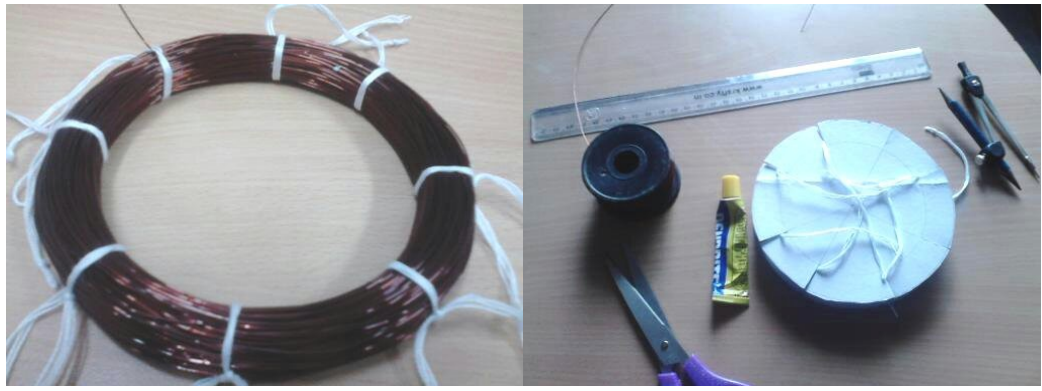

Figure 6: Magnetic coil and bobbin after construction.

Fig. 6 shows the magnetic coil and the bobbin after construction. A 26 gauge super enameled wire is winded around the bobbin for 1500 times. After winding is done, the bobbin is dismantled. The whole coil is then insulated by PVC 1/2" tape. Two multi-strand long copper wires were used to connect the lead wire of the magnetic coil by soldering them. Below table lists the physical parameters of the developed magnetic coil and bobbin.

TABLE-1

\begin{tabular}{|l|l|c|c|}
\hline SI No. & \multicolumn{1}{|c|}{ Parameter } & Values & Unit \\
\hline 1 & Outer radius of coil (r1) & 110 & $\mathrm{~mm}$ \\
\hline 2 & Inner radius of coil (r2) & 80 & $\mathrm{~mm}$ \\
\hline 3 & Thickness (x2-x1) & 22 & $\mathrm{~mm}$ \\
\hline 4 & No of turns (n) & 1500 & --- \\
\hline 5 & Super Enameled copper wire gauge & 26 & SWG \\
\hline 6 & Insulation of wire & Double layered & --- \\
\hline 7 & Lead wire & PVC flexible & Multi Strand mt. \\
\hline 8 & Termination (Male jack) & 4 & Male jack \\
\hline 9 & Overall insulation & Teflon & --- \\
\hline
\end{tabular}

The electrical specifications of the developed coil were tested using RLC Q-meter. Fig 7 shows the test setup for the same. 


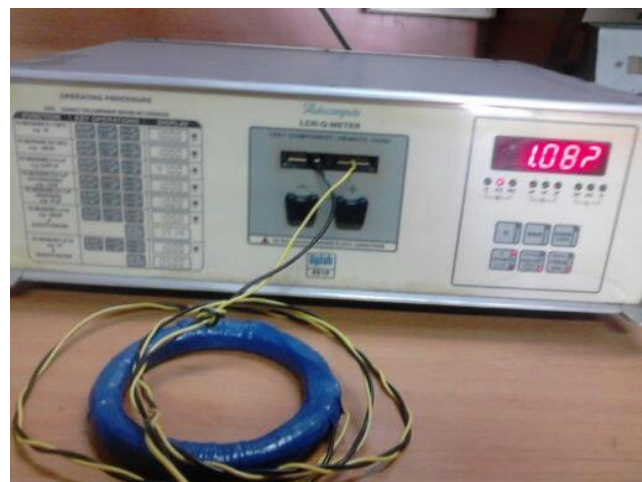

Figure 7: Testing of Coil using RLC Q-model 9410.

The results of the electrical tests are as given below:

TABLE-2

\begin{tabular}{|l|l|l|}
\hline SI No. & Parameter & Values \\
\hline 1 & DC resistance & 135.7 \\
\hline 2 & Inductance & 98.3 \\
\hline 3 & Magnetic field (B) at $0.55 \mathrm{amps}$ & 125.35 \\
\hline
\end{tabular}

\subsubsection{DESIGN OF MICROCONTROLLER BASED MAGNETIC COIL DRIVER}

To drive and power the magnetic coil L298 we have used a dual full bridge driver integrated circuit. It is a high voltage, high current, dual full bridge driver designed to accept standard TTL logic. Special care has also been taken to protect the circuit. A LM339 comparator is used to protect this circuit from overload and short circuit. Fig. 8 shows the complete circuit of microcontroller based magnetic coil driver.

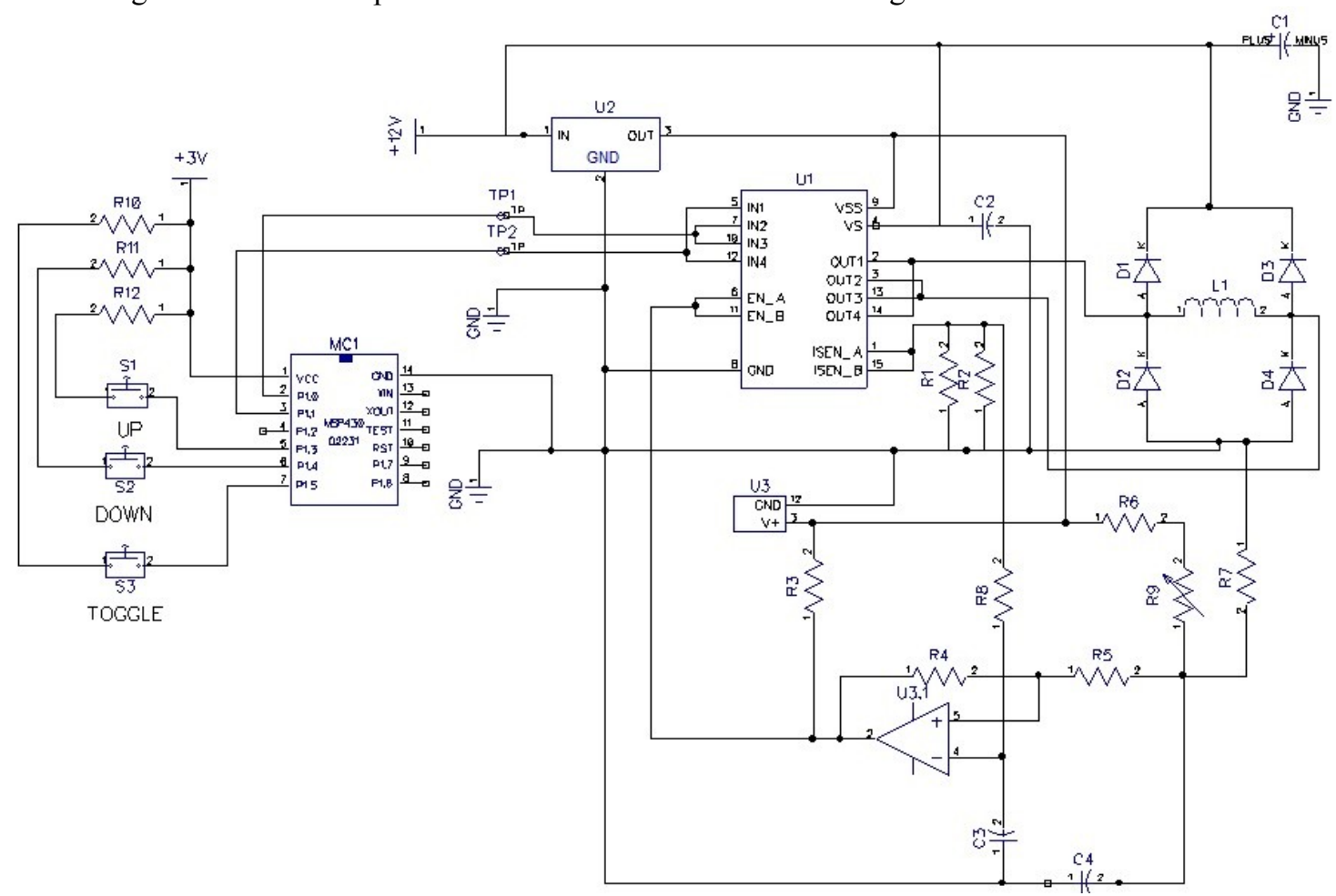

Figure 8: Circuit diagram of microcontroller based Electro Magnetic Pulse therapy instrument.

MSP430G2231 has been used [11] to generate pulses with variable frequency for the full bridge in power drive circuit. MSP430G2231 is an ultra-low power controlled micro controller featuring various features targeting multifarious applications. The architecture combines with five low power modes and features a powerful 16 bit RISC CPU, 16 bit registers, and constant generators that contribute to maximum code efficiency. The digitally controlled oscillator (DCO) allows wake up from low power modes to active mode in less than $1 \mu \mathrm{s}$. It has inbuilt 16-bit timer with two capture/compare registers. It also has a 10-Bit $200 \mathrm{ksps} \mathrm{A} / \mathrm{D}$ 
converter with internal reference, sample-and-hold, and auto scan features. MSP4302231 has built in serial on board programming capabilities.

Before we place the microcontroller in the circuit as shown in Fig 8, we need to program it such that it we can control frequency and output pin. Pin P1.3 is the interrupt pin for UP signal and Pin P1.4 is the interrupt pin for down signal. The program is written in a way such that we can control frequency in 30 precise steps. There are two output pins P1.0 and P1.1 which can be toggled by passing in interrupt at pin P1.5. To program the MSP430G2231 we used MSP430 Launch Pad [10] which is a microcontroller development kit from Texas Instruments. The MSP430 Launchpad has an onboard flash emulator, USB, 2 programmable LEDs, and 1 programmable push button. Once connected to computer via USB we can use IDE like Code Composer Studio to code and burn the programs in the micro controller. Fig. 9 illustrates the flowchart of pulse generator with variable frequency using MSP430G2231.The codes are written in Microcontroller C programming language. Fig. 9 shows the flowchart of the embedded program.
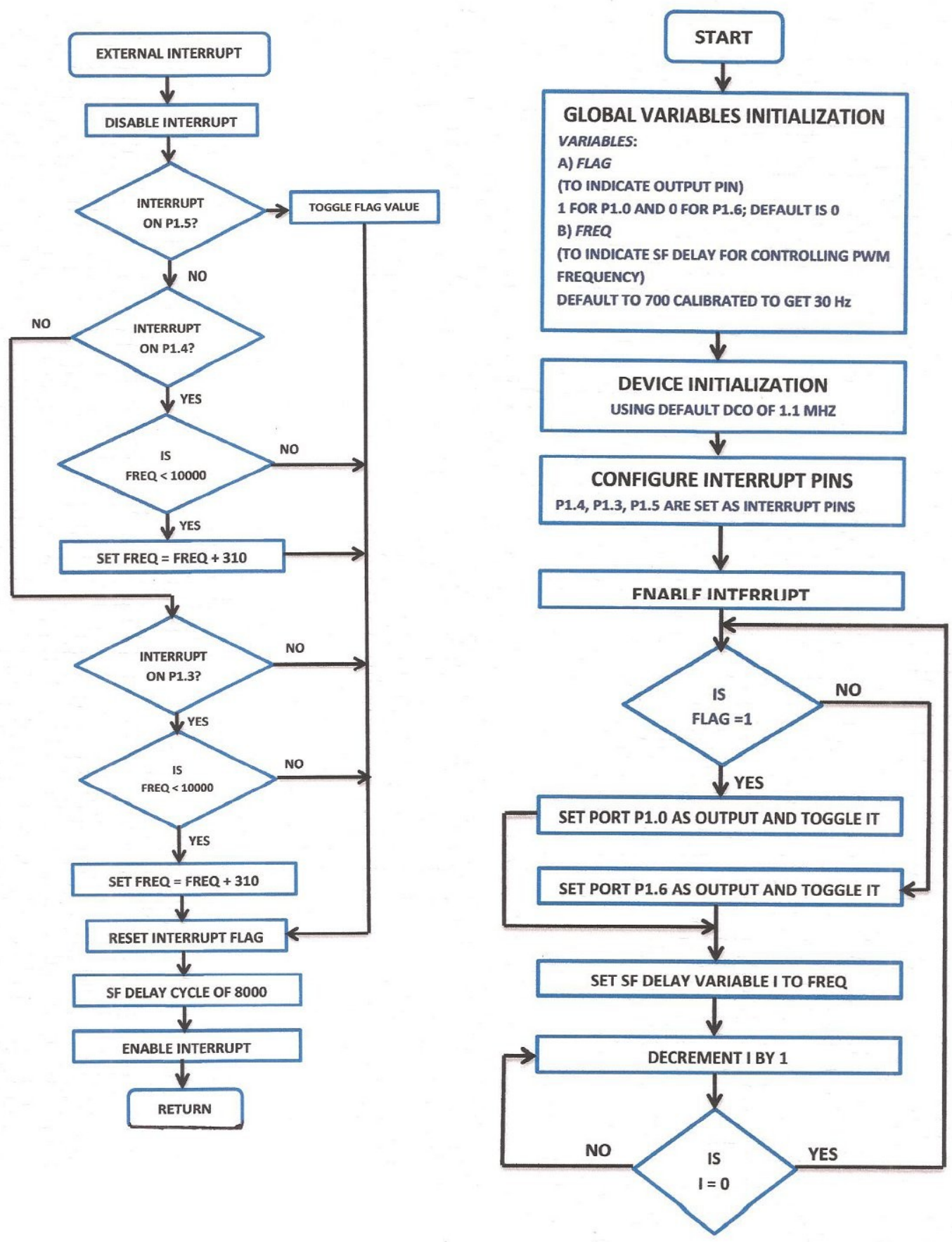

Figure 8: Flowchart of microcontroller embedded software. 


\subsection{FABRICATION, CALIBRATION AND TESTING OF PROTOTYPE}

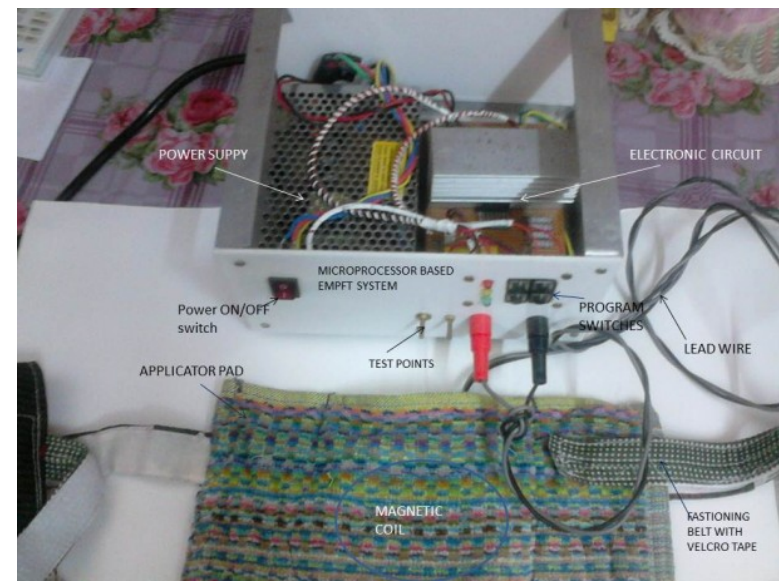

Figure 9: Fabricated prototype of PEMFT device.

The design of printed circuit board was made using Diptrace PCB CAD software, all electronic components were soldered. After completing the fabrication of PCB has been tested with Digital storage oscilloscope and frequency of the pulse has been calibrated with the DSO through modification in the software routine. It is assembled in a custom made enclosure as shown in Fig.9. The magnetic coil is kept inside cotton pad with Velcro strip. The lead wire is about 1.5 meter long. The magnetic coil with pad can be place over the part of the body and can be fastened by the Velcro tape. The leads of magnet ended with $4 \mathrm{~mm}$ connecting pin. Frequency and toggle program switches are located in the front panel of the equipment.

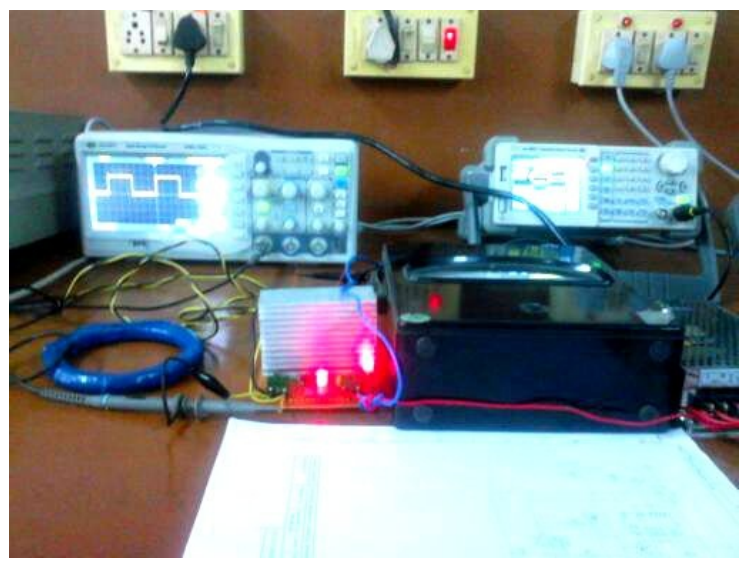

Figure 10: Calibration and testing of PEMFT device.

\section{Results And Discussion}

Three human male volunteers having low back pain had been selected for initial field trial to find out the performance of the equipment. The frequency of the EMPTF was set to $10 \mathrm{~Hz}$. No additional medicine, physiotherapy massages or pain relief ointments were applied to the volunteers. Only 30 minutes x 14 days therapy with the prototype was applied to the volunteers. The observed pain relief was satisfactory. However study with three volunteers is not significant enough for clinical practice. More long term clinical studies with more number of volunteers are required to get Clinical acceptance. Calibration and repeatability of the electrical parameters were also checked with digital storage oscilloscope and was found to be satisfactory

The equipment needs further development like inclusion of frequency display unit to measure the frequency of applied electromagnetic pulse. More fine frequency control can be achieved implementing more frequency steps in the software. Further improvement of magnetic coil design can be done such that a more accurate magnetic field can be applied to the point of application without heating.

Cost of building the prototype is around $3000 \mathrm{INR}$, which is quite less when compared to the products commercially available in market. Pulse Electromagnetic therapy devices are not very popular in India. No indigenous version are available here, therefore there is a good possibility of developing low cost pulse electromagnetic therapy system as part of consumer electronics. 


\section{References}

[1]. William Pawluk, "Pulsed Electromagnetic Fields: How They Heal", http://www.doctoroz.com/videos/pulsed-electromagneticfields-how-they-heal

[2]. Bassett CA, Schink-Ascani M, Lewis SM. (September 1989). "Effects of Pulsed Electromagnetic Fields on Steinberg Ratings of Femoral Head Osteonecrosis", Clin Orthop Relat Res. 1989 Sep;(246):172-85

[3]. Markov, Marko S (2007). "Expanding Use of Pulsed Electromagnetic Field Therapies". Electromagnetic Biology \& Medicine 26 (3): 257-274. doi:10.1080/15368370701580806

[4]. PRJVC Boopalan, Samuel B. Chittaranjan, Ramadass Balamurugan, N.S. Nandakumar, A. Sabareeswaran and Mira Mohanty, "Pulsed electromagnetic field (PEMF) treatment for fracture healing". Current Orthopaedic Practice 01/2009; 20. DOI: $10.1097 / \mathrm{BCO} .0 \mathrm{~b} 013 \mathrm{e} 318198 \mathrm{e} 8 \mathrm{~b} 2$

[5]. Mark Sircus, "Pulsed Electromagnetic Field, Therapy for Cancer and Pain”, December 28, 2012 | Filed under Cancer, Medicine, http://drsircus.com/medicine/cancer/pulsed-electromagnetic-field-therapy-cancer-pain

[6]. Pulsed electromagnetic field therapy - Wikipedia, the free encyclopedia, http://en.wikipedia.org/wiki/Pulsed_electromagnetic_field_therapy

[7]. Walter H. Chang, Jimmy K. Li, James Cheng-An Lin, Hwa-Chang Liu and Jui-Sheng Sun,'Bone Defect Healing Enhanced by Pulsed Electromagnetic Fields Stimulation: in Vitro Bone Organ Culture Model" , Journal of Medical and Biological Engineering, 25(1), 2005, 27-32

[8]. Satter Syed A, Islam MS, Rabbani KS, Talukder MS," Pulsed electromagnetic fields for the treatment of bone fractures." , Bangladesh Med Res Counc Bull. 1999 Apr;25(1):6-10.

[9]. MSP430G2x21, MSP430G2x31 Mixed Signal Microcontroller (Rev. J), Texas Instruments,

[10]. http://www.ti.com/product/msp430g2231

[11]. MSP430 LaunchPad Value Line Development kit, Texas Instruments, http://www.ti.com/tool/msp-exp430g2

[12]. L298Dual Full Bridge Driver, ST Microelectronics,

http://www.st.com/web/en/catalog/sense_power/FM142/CL851/SC1790/SS1555/PF63147

[13]. LM339 Quad Comparator, Fairchild Semiconductor, https://www.fairchildsemi.com/pf/LM/LM339.html 